\title{
Edema-informed anatomically constrained particle filter tractography
}

\author{
Samuel Deslauriers-Gauthier ${ }^{1}$, Drew Parker $^{3}$, François Rheault ${ }^{2}$, Rachid \\ Deriche $^{1}$, Steven Brem ${ }^{3}$, Maxime Descoteaux ${ }^{2 \star}$, and Ragini Verma ${ }^{3 \star}$ \\ ${ }^{1}$ Inria Sophia Antipolis, Université Côte d'Azur, France \\ ${ }^{2}$ Université de Sherbrooke, Sherbrooke, Canada \\ ${ }^{3}$ University of Pennsylvania, Philadelphia, USA
}

\begin{abstract}
In this work, we propose an edema-informed anatomically constrained tractography paradigm that enables reconstructing larger spatial extent of white matter bundles as well as increased cortical coverage in the presence of edema. These improvements will help surgeons maximize the extent of the resection while minimizing the risk of cognitive deficits. The new paradigm is based on a segmentation of the brain into gray matter, white matter, corticospinal fluid, edema and tumor regions which utilizes a tumor growth model. Using this segmentation, a valid tracking domain is generated and, in combination with anatomically constrained particle filter tractography, allows streamlines to cross the edema region and reach the cortex. Using subjects with brain tumors, we show that our edema-informed anatomically constrained tractography paradigm increases the cortico-cortical connections that cross edemacontaminated regions when compared to traditional fractional anisotropy thresholded tracking.
\end{abstract}

\section{Introduction}

Diffusion magnetic resonance imaging (MRI) allows to non-invasively reconstruct white matter tracts. In the context of pre-operative surgical planning, tractography is used to identify and locate fiber bundles in the vicinity of the lesion. Equipped with this knowledge, the surgeon attempts to maximize tumor removal while minimizing damage to functional white matter networks [1]. While the development efforts on every step of the tractography pipeline are extensive, most algorithms assume healthy brain and are tested on healthy white matter models. Diffusion tensor imaging tractography has been investigated in the context of neurosurgery $[5,6]$, but is subject to the well known limitation of the diffusion tensor. There is thus very little data on the performance of modern tractography algorithms in the presence of edematous or tumoral tissue. One notable exception is the use of multi-tensor models in the context of unscented Kalman filter (UKF) tractography, which has been investigated on brain tumor patients [2-4]. The authors show that two-tensor UKF increases the volume of the arcuate fasciculus and corticospinal tract and their coverage of the functional MRI

* Co-senior authors. They have contributed equally. 

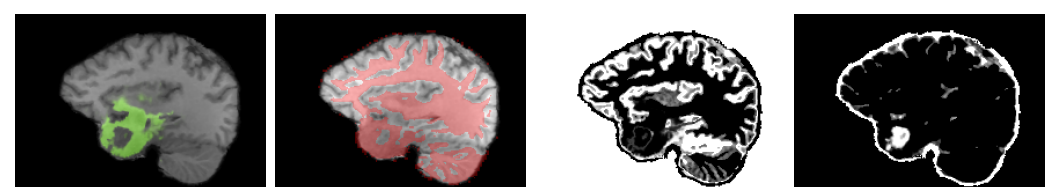

Fig. 1. Segmentation of the edema obtained from GLISTR (first) and the tracking mask obtained by thresholding the FA at 0.2 (second). The edema lowers the FA and isolates the temporal lobe. The include map (third) and exclude map (fourth) show that in EI-PFT the edema can be traversed as it is in black in both masks. The tumor is visible in the exclude map indicating that streamlines cannot end in this region.

activation sites, especially when used with whole brain seeding. These results highlight the need for further investigation into the use of modern tractography strategies for pre-operative surgical planning.

Tractography algorithms reconstruct streamlines in the white matter of the brain by following the local diffusion directions obtained through diffusion MRI. While several criteria can be used to stop the tracking, the most common is to place a threshold on the fractional anisotropy (FA) map [7]. The rational is that the FA is high in regions with coherent fiber directions and therefore acts as a proxy for the white matter. However, it is well known that many phenomena can lower the measured FA, such as fiber crossings, free water contamination, and pathologies like edema in the presence of tumor [9]. In these scenarios, the tracking may be prematurely terminated yielding a streamline that does not reach the cortex. Furthermore, because the FA does not encode anatomical information, algorithms that rely on it will produce physically impossible streamlines, such as those that start or end in the ventricles. Anatomically constrained tractography $(\mathrm{ACT})[8]$ alleviates this problem by defining a valid tracking domain based on the anatomy of the subject defined by the tissue segmentation. In combination with particle filter tractography, which backtracks and attempts to find alternate routes when it prematurely hits a region outside of the valid tracking domain, $\mathrm{ACT}$ was shown to reduce the number of false positive streamlines [10].

Because edema and tumor change the local tissue properties of the brain, tractography based on FA thresholding may not provide an accurate representation of fiber near the lesion. In this paper, we provide a paradigm that integrates anatomical information of both healthy and unhealthy tissues with particle filter tracking to obtain tractography through edema. This has been demonstrated on 4 tumor subjects.

\section{Methods}

\subsection{Edema-informed anatomically constrained particle filter tractography}

Tracking is rendered edema-informed by defining a valid tracking domain which integrates the knowledge of the various tissue types: white matter, gray matter, 


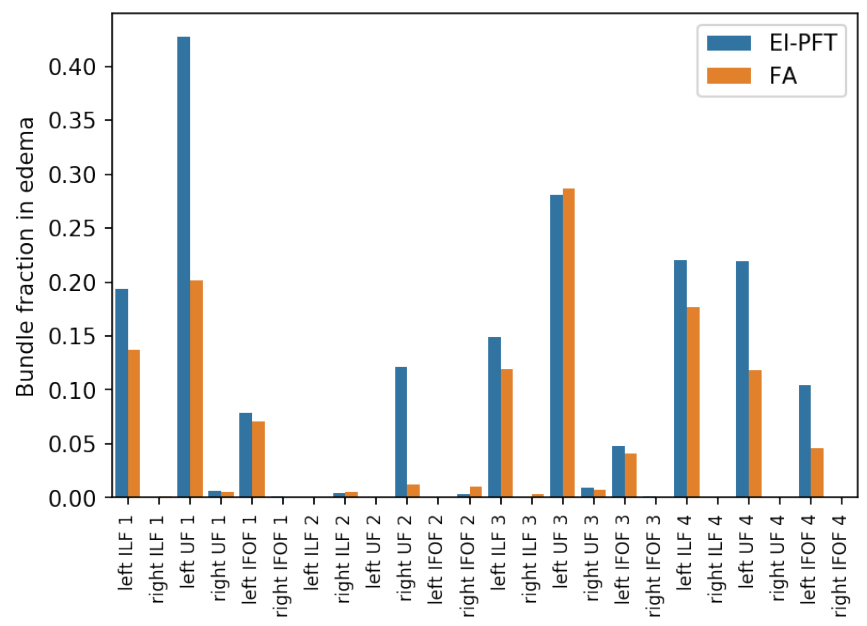

Fig. 2. Comparison of the bundle fraction in edema obtained using EI-PFT or FA threshold tractography. Larger values indicates that a greater proportion of the bundle volume intersects with the edema.

corticospinal fluid, edema, and tumor segmentation. More specifically, the tracking domain is defined by 3 probabilistic maps. The first map, referred to as the include map, determines valid start and end points for streamlines. That is, the value of a voxel of the include map gives the probability that a streamline will terminate within this voxel and be included in the final tractogram. An example of an include map is illustrated in the third image of Figure 1 where white regions indicate valid streamline start or end points. The second map, referred to as the exclude map, determines the probability that a streamline will terminate within a region and be excluded from the final tractogram. An example of an exclude map is illustrated in the fourth image of Figure 1 where white regions indicates invalid streamlines start or end points. Note that for any given voxel, the sum of the two maps must be between zero and one. Together, the include and exclude maps define a third implicit map which ensure the probabilities sum to one. This third map contains the regions that streamlines are free to cross, but where they cannot terminate. This separation of the tracking domain into 3 maps was used in [10], but their definition of the maps was based solely on the segmentation of healthy tissues, whereas we include edema and tumor information. Specifically, we define the include map to be the gray matter segmentation and the exclude map as $P_{e x}=P_{\text {tumor }}+P_{c s f}-P_{\text {tumor }} P_{c s f}$ where $P_{\text {tumor }}$ and $P_{c s f}$ are the tumor and CSF segmentations, respectively. Note that the edema segmentation map is not used explicitly in the computation of the tracking domain, but it nonetheless affects it implicitly. Indeed, just like the white matter, the edema is considered as a region where the streamlines can traverse but not end. These changes to the tracking domain have two important effects on the final tractogram. First, streamlines entering the tumor will now be excluded, as the 

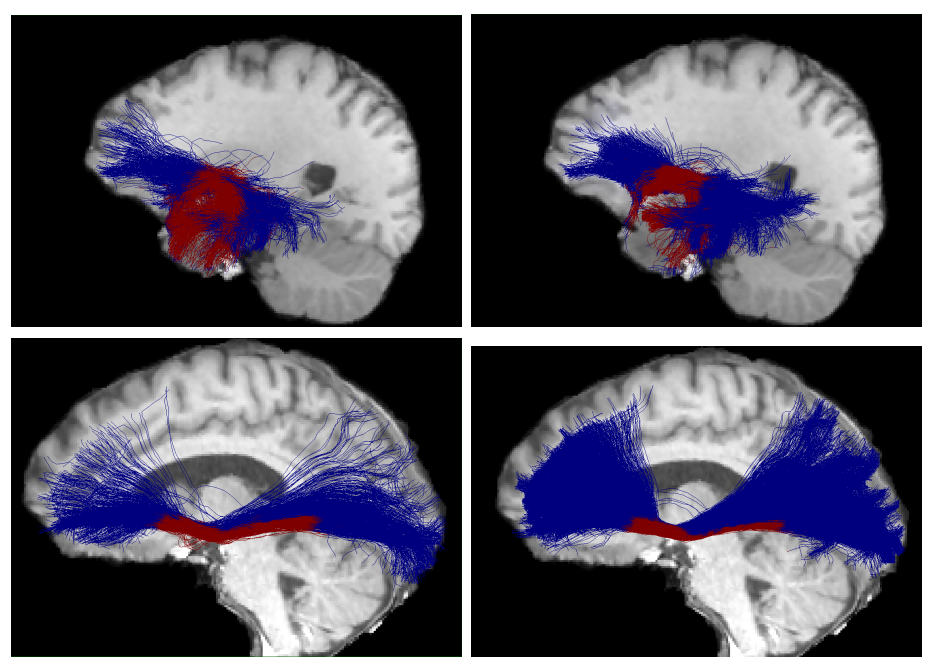

Fig. 3. Comparison of the UF of subject 1 (top) and the IFOF of subject 7 (bottom) reconstructed using EI-PFT (left) and FA thresholded tractography (right). For subject 1 , the streamlines recovered by EI-PFT penetrate further into the temporal lobe and wrap the tumor. This is consistent with the maps of Figure 1 where the temporal lobe was isolated by the lowered FA of the edema. For subject 4, the streamlines projecting to the inferior portion of the occipital lobe have a greater intersection with the edema when reconstructed with IE-PFT.

tumor is part of the exclude map. Second, streamlines displaced by the tumor are more likely to be identified because the particle filter will backtrack and attempt to find a way around the tumor in the edema. We refer to this method as edema-informed anatomically constrained particle filter tractography (EI-PFT). It should be noted that common segmentation tools, e.g. FAST (FSL), assume a healthy brain and will therefore misclassify edematous or tumoral tissue as white matter, gray matter, or corticospinal fluid. These misclassifications are of critical importance in our paradigm because they directly influence the tracking domain and thus the final tractogram. For example, considering edematous tissue as gray matter will cause streamlines to prematurely terminate inside the lesion and yield an erroneous connection. Likewise, misclassifying a tumor may allow streamlines to traverse the tumor instead of being displaced by it. To produce the final segmentation, we therefore opted for the fusion of two segmentations. First, the white matter, gray matter, and corticospinal fluid segmentations are based on the partial volumes obtained with FAST (FSL) as it is very reliable in healthy regions away from the lesion. Second, the edema and tumor regions are based on the glioma image segmentation and registration (GLISTR) [11] tool which provides accurate tumor and edema segmentation based on tumor growth modeling. To ensure the maps of the 5 labels sum to one, the corrected maps were computed as $P_{*}=\bar{P}_{*}-P_{\text {edema }} \bar{P}_{*}-P_{\text {tumor }} \bar{P}_{*}$ where $P_{\text {edema }}$ and $P_{\text {tumor }}$ are 


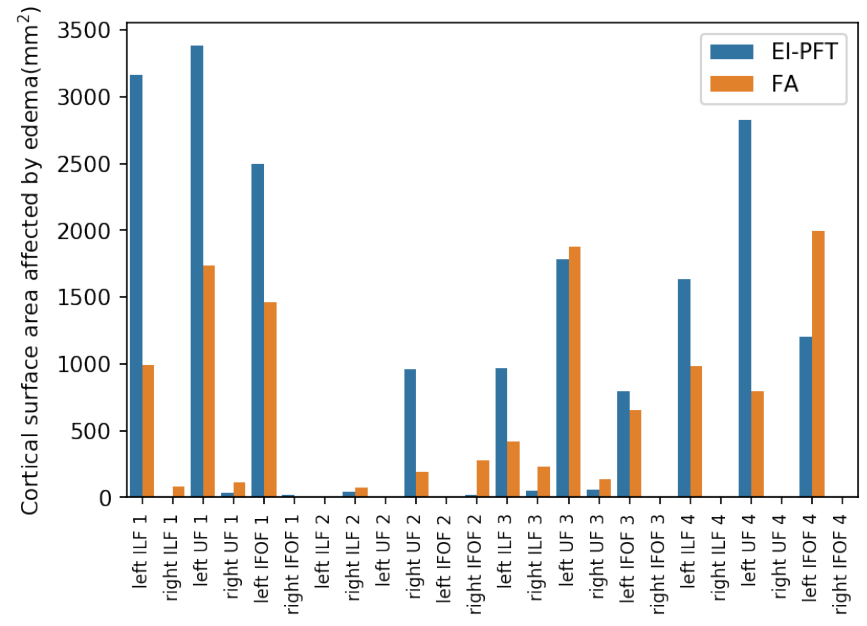

Fig. 4. Comparison of the cortical surface area where streamlines intersecting the edema terminate recovered by EI-PFT and FA thresholding tractography. Larger values indicate that more streamlines traverse the edema and reach the cortex.

GLISTR outputs and $\bar{P}_{*}$ is the white matter, gray matter, or corticospinal fluid map obtained from FAST.

\subsection{Data acquisition and preprocessing}

For 4 subjects having a tumor near the temporal stem, multi-shell multi-band DWI $[\mathrm{TR} / \mathrm{TE}=5,216 / 100 \mathrm{~ms}$, resolution $=2 \mathrm{~mm}$ isotropic, 64 diffusion directions at $\mathrm{b}=2,000 \mathrm{~s} / \mathrm{mm}^{2}, 30$ diffusion directions at $\mathrm{b}=800 \mathrm{~s} / \mathrm{mm}^{2}, 15$ diffusion directions at $\mathrm{b}=300 \mathrm{~s} / \mathrm{mm}^{2}$, and $7 \mathrm{~b}=0$ images] were acquired on an Siemens 3T TrioTim scanner using a 32-channel head coil. In a separate session on a Siemens 3T TrioTim, T1 MPRAGE, T1 contrast-enhanced (0.977 x $0.977 \times 1 \mathrm{~mm})$, T2 and FLAIR images were acquired using a 12-channel head coil. The diffusion MRI volumes were corrected for distortion correction and motion correction using eddy (FSL). Structural images were bias corrected with N3 (ITK), smoothed with susan (FSL) and then coregistered to T1 with flirt (FSL), and then all were brought to FA and b0 space with ANTs. The fiber orientation distribution functions were then computed using constrained spherical deconvolution using a spherical harmonic order of 8. FSL FAST was used to segment the T1 image into white matter, gray matter, and corticospinal fluid partial volume maps. GLISTR was used on the T1, T2, T1 contract-enhanced, and FLAIR to segment the lesion into edema and tumor regions. The output of FAST and GLISTR were used to define the include and exclude maps of the anatomically constrained tractography, as described in the previous section. An example of the include and exclude maps obtained for subject 1 are illustrated in Figure 1. Probabilistic particle filter tractography was then used to generate 

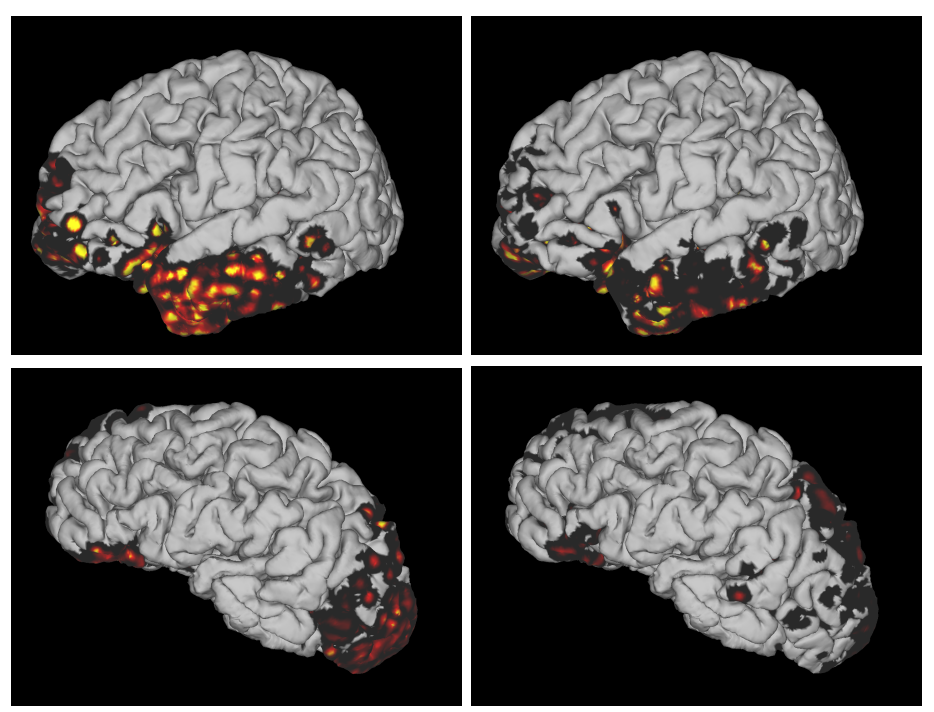

Fig. 5. The cortical surface area of streamlines intersecting the area obtained using EIPFT (left) and FA thresholded tractography (right) for the UF of subject 1 (top) and the IFOF of subject 4 (bottom). Warmer color indicates that more streamlines that intersect the edema terminate in that region of the cortex. EI-PFT has more intense and broader areas in the the temporal and frontal poles, indicating areas that may still be functionally active even if they are affected by the lesion.

the streamlines by using 1 seed per voxel over the white matter mask. Finally, the streamlines were automatically segmented into bundles using recobundles [12], which makes use of streamline geometry instead of anatomical landmarks. This streamlines segmentation strategy is critical as brain tumor subjects may have severe deformations which prevent typical landmarks from being identified. Because our subjects all have a tumor near the temporal stem, we report the results for the inferior longitudinal fasciculus (ILF), uncinate fasciculus (UF), and the inferior fronto-occipital fasciculus (IFOF). These 3 bundles all funnel near the temporal stem and were the most affected by the lesion. To evaluate if EI-PFT is able to traverse edema regions, we also report result obtained by using FA thresholded tractography pipeline. The same fiber orientation distribution functions were used as input to probabilistic tractography where the tracking was stopped when the FA dropped below 0.2.

\section{Results}

To quantify our results, we computed the volume of the recovered bundles that intersect the edema mask. To obtain normalized values, we divide this volume by the total volume of the bundle, yielding a bundle fraction in edema. Since the volume of a bundle can be affected by shorter streamlines, we also compute 
the cortical surface area overlapping with the streamlines that traverse through the edema and reach the cortical surface. This surface area was estimated by selecting the streamlines that intersect the edema and by counting the number of start and end voxels of each streamline that intersect with the cortical surface. For both the bundle fraction in edema and the cortical surface area of streamlines intersecting the edema, we expect larger values to be obtained by tractography pipelines are able to penetrate edematous regions.

Figure 2 illustrates the bundle fraction in edema for each of our bundles of interest. Out of 9 bundles with a non-negligible bundle fraction in edema (above 5\%), 8 had a larger fraction using EI-PFT than FA thresholding tractography. These results are notable because the complete paradigm includes an automatic streamline bundling step that removes streamlines that are not detected in bundles. In other words, EI-PFT explores the edematous region more than FA thresholding while still producing streamlines that are classified as part of a bundle. This is further illustrated in Figure 3, where the uncinate fasciculus of subject 1 is illustrated. It is clear that both EI-PFT and FA thresholding tractography produce 'bundle like' outputs, but EI-PFT has a greater intersection with the edema. Figure 4 compares the cortical surface area of streamlines intersecting the edema obtained using EI-PFT and FA thresholding tractography. Out of 10 bundles with a non-negligible area (above $500 \mathrm{~mm}^{2}$ ), 8 have a larger area when obtained using EI-PFT. This highlights thats EI-PFT explores the edematous tissue while producing cortico-cortical streamlines. Examples of these surfaces are presented in Figure 5. The images were generated by coloring the cortical endpoints of the streamlines by their overlap with the edema mask. It can be observed that EI-PFT yields broader and more intense cortical surface area indicating regions where streamlines overlapping the edema terminate.

\section{Conclusion}

The surgical treatment of any intrinsic brain pathology necessitates careful planning to develop a safe trajectory from the cortical surface, through sulci and white matter to reach the tumor boundary while minimizing injury to the white matter tracts. Our evaluation demonstrates that EI-PFT is able to better achieve this in comparison to the FA thresholded tractography that is available in the surgical planning tools, potentially leading to safer resections. Additionally, larger tract volume in the edema, which our paradigm is able to achieve due to tracking through edema, is a precursor to increased margins of resection and hence better patient outcome.

\section{Acknowledgements}

This work was supported by NIH grant R01NS096606 (PI: Verma, Brem) and has received funding from the European Research Council (ERC) under the European Union's Horizon 2020 research and innovation program (ERC Advanced Grant agreement No 694665: CoBCoM). 


\section{References}

1. M. J. McGirt, K. L. Chaichana, M. Gathinji, F. J. Attenello, K. Than, A. Olivi, J. D. Weingart, H. Brem, and A. R. Quinones-Hinojosa, "Independent association of extent of resection with survival in patients with malignant brain astrocytoma", J. Neurosurg., vol. 110, pp. 156-162, 2009.

2. Z. Chen, Y. Tie, O. Olubiyi, L. Rigolo, A. Mehrtash, I. Norton, O. Pasternak, Y. Rathi, A. J. Golby, and L. J. O'Donnell, "Reconstruction of the arcuate fasciculus for surgical planning in the setting of peritumoral edema using two-tensor unscented Kalman filter tractography", NeuroImage: Clinical, vol. 7, pp. 815-822, 2015.

3. Z. Chen, Y. Tie, O. Olubiyi, F. Zhang, A. Mehrtash, L. Rigolo, P. Kahali, I. Norton, O. Pasternak, Y. Rathi, A. J. Golby, L. J. O'Donnell, "Corticospinal tract modeling for neurosurgical planning by tracking through regions of peritumoral edema and crossing fibers using two-tensor unscented Kalman filter tractography", Int. J. CARS, vol. 11, pp. 1475-1486, 2016.

4. R. Liao, L. Ning, Z. Chen, L. Rigolo, S. Gong, O. Pasternak, A. J. Golby, Y. Rathi, and L. J. O'Donnell, "Performance of unscented Kalman filter tractography in edema: Analysis of the two-tensor model", NeuroImage: Clinical, vol. 15, pp. 819-831, 2017.

5. M. Mandelli, M.S. Berger, M. Bucci, J. Berman, B. Amirbekian, and R.G. Henry. "MandeliQuantifying accuracy and precision of diffusion MR tractography of the corticospinal tract in brain tumors", J Neurosurg, vol. 121, pp.349-358, 2014.

6. S. Pujol, W. Wells, C. Pierpaoli, C. Brun, J. Gee, G. Cheng, B. Vemuri, O. Commowick, S. Prima, A. Stamm, M. Goubran, A. Khan, T. Peters, P. Neher, K.H. Maier-Hein, Y. Shi, A. Tristan-Vega, G. Veni, R. Whitaker, M. Styner, C.F. Westin, S. Gouttard, I. Norton, L. Chauvin, H. Mamata, G. Gerig, A. Nabavi, A. Golby, and R. Kikinis. "The DTI Challenge: Towards Standardized Evaluation of Diffusion Tensor Imaging Tractography for Neurosurgery", J Neuroimaging, vol. 25, pp. 875-882, 2015.

7. B. Jeurissen, M. Descoteaux, S. Mori, A. Leemans, "Diffusion MRI fiber tractography of the brain", NMR in biomedicine, 2017.

8. R. E. Smith, J. D. Tournier, F. Calamante, A. Connelly, "Anatomically-constrained tractography: Improved diffusion MRI streamlines tractography through effective use of anatomical information", Neuroimage, vol. 62, pp. 1924-1938, 2012.

9. H. Duffau, "The dangers of magnetic resonance imaging diffusion tensor tractography in brain surgery", World neurosurgery, vol. 81, pp. 56-58, 2014.

10. G. Girard, K. Whittingstall, R. Deriche, and M. Descoteaux, "Towards quantitative connectivity analysis: reducing tractography biases", NeuroImage, vol. 98C, pp. 266$278,2014$.

11. A. Gooya, K. M. Pohl, M. Bilello, L. Cirillo, G. Biros, E. R. Melhem, and C. Davatzikos, "GLISTR: Glioma image segmentation and registration", IEEE Transations on medical imaging, vol 31, pp. 1941-1954, 2012.

12. E. Garyfallidis, M. A. Côté, F. Rheault, J. Sidhu, J. Hau, L. Petit, S. Cunanne, and M. Descoteaux, "Recognition of white matter bundles using local and global streamline-based registration and clustering", NeuroImage, 2017. 